\title{
Accident vasculaire cérébral ischémique à IRM normale
}

\author{
False-Negative MRI in Acute Ischemic Stroke
}

\author{
N. Bergot · J. Kosowski · P.C. Thiebaud \\ Reçu le 22 mars 2018; accepté le 14 mai 2018 \\ (C) SFMU et Lavoisier SAS 2018
}

\section{Introduction}

Le diagnostic précoce des accidents vasculaires cérébraux (AVC) ischémiques repose actuellement sur la séquence de diffusion de l'imagerie par résonance magnétique (IRM) cérébrale. Malgré son excellente sensibilité, il existe des faux négatifs. Nous présentons le cas d'une patiente présentant un AVC ischémique constitué malgré une IRM cérébrale initiale normale.

\section{Cas clinique}

Une patiente de 67 ans est transportée aux urgences suite à un malaise sans perte de conscience au décours duquel sont constatés par son entourage une confusion et un manque du mot. Ses principaux antécédents sont une cardiopathie ischémique stentée, un diabète insulinorequérant compliqué d'une insuffisance rénale terminale en dialyse péritonéale quotidienne, une hypertension artérielle et une hypercholestérolémie. À son arrivée aux urgences, la patiente a une conscience normale mais présente une aphasie totale. Le reste de l'examen neurologique est normal, il n'existe pas de déficit moteur évident des membres ni de paralysie faciale. Le score NIHSS est à 7. L'hémodynamique est stable, la patiente est eupnéique, apyrétique, et la glycémie capillaire est à $14,4 \mathrm{mmol} / \mathrm{l}$. L'électrocardiogramme montre un rythme sinusal régulier, un bloc de branche gauche connu, sans trouble significatif de repolarisation. Une IRM cérébrale, réalisée en urgence à $160 \mathrm{~min} \mathrm{du}$ début des symptômes dans le cadre d'une alerte thrombolyse, ne retrouve pas d'argument pour une lésion ischémique ou hémorragique récente (Fig. 1A). Le polygone de Willis est complet et perméable, il existe une sténose athéromateuse non significative des carotides internes dans leur portion intra-

\footnotetext{
N. Bergot $\cdot$ J. Kosowski $\cdot$ P.C. Thiebaud $(\bowtie)$

Service des urgences, hôpital Tenon,

Assistance publique-Hôpitaux de Paris (AP-HP),

4, rue de la Chine, F-75020 Paris, France

e-mail : pc.thiebaud@hotmail.fr
}

caverneuse et un aspect grêle de l'artère vertébrale gauche. Le bilan biologique ne retrouve pas d'anomalie métabolique. Après avis neurovasculaire, il est décidé de réaliser un électroencéphalogramme (EEG) à la recherche d'une épilepsie partielle. L'EEG ne montre pas de signes de comitialité, mais une souffrance dans la région frontotemporale gauche évoquant une cause lésionnelle et compatible avec un AVC. Une relecture de l'IRM initiale est alors demandée au neurovasculaire et au radiologue, qui confirment l'absence d'AVC visible. La patiente reçoit $250 \mathrm{mg}$ d'aspirine et est hospitalisée

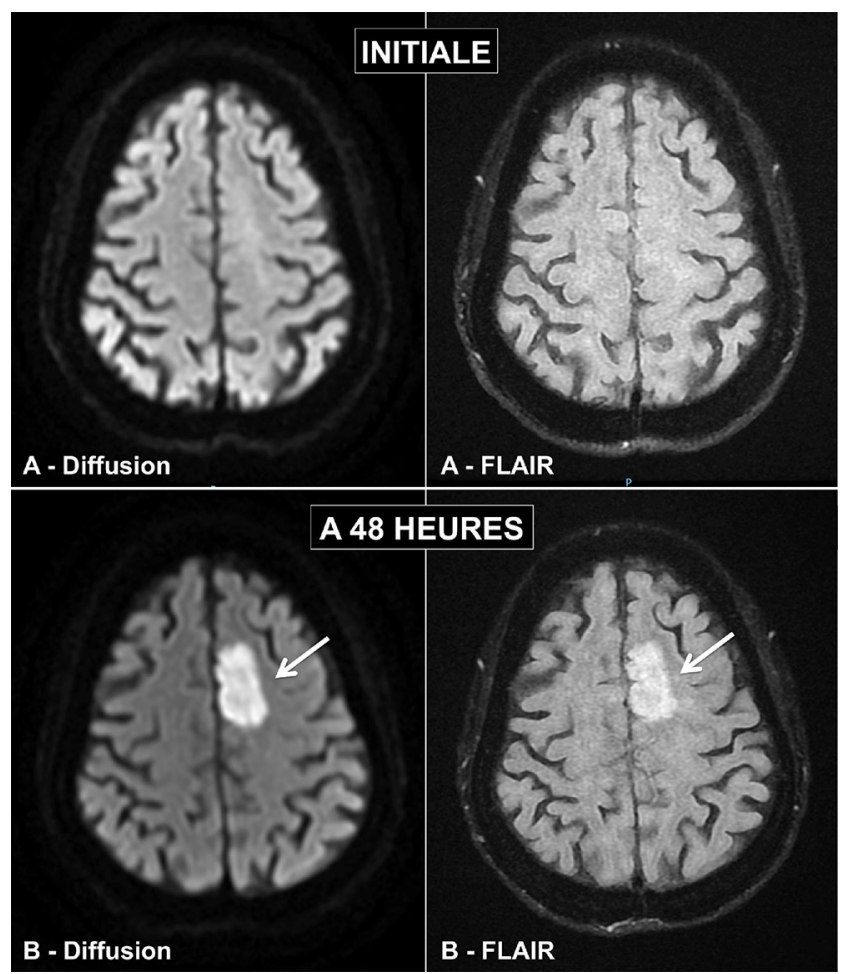

Fig. 1 Imageries par résonance magnétique (IRM) cérébrales en séquences de diffusion et FLAIR. L'IRM initiale (A) ne montre pas d'anomalie significative. L'IRM de contrôle à 48 heures (B) retrouve un hypersignal dans le territoire de l'artère cérébrale antérieure gauche (flèches), en relation avec un accident vasculaire cérébral ischémique récent 
en soins intensifs de néphrologie. Devant la persistance de l'aphasie, une nouvelle IRM cérébrale est réalisée 48 heures plus tard (Fig. 1B). Elle retrouve une ischémie récente dans le territoire superficiel de l'artère cérébrale antérieure gauche. Le diagnostic d'AVC ischémique constitué est alors posé, et la patiente transférée en unité de soins intensifs neurovasculaires.

\section{Discussion}

La séquence de diffusion de l'IRM cérébrale est actuellement l'examen de référence dans le diagnostic précoce des AVC ischémiques [1,2] grâce à son excellente sensibilité de 89 à $100 \%$ [3]. Cependant, plusieurs cas d'AVC ischémiques à IRM cérébrale initiale normale ont été décrits dans la littérature, cette situation représenterait entre 1,5 et $9,5 \%$ des cas [4-8]. D'après Ay et al., parmi les patients présentant un déficit neurologique suspect et une IRM cérébrale initiale normale, un diagnostic d'AVC ischémique constitué est posé devant l'apparition secondaire d'une anomalie radiologique dans $25 \%$ des cas [4]. Les facteurs de risque de ces faux négatifs décrits dans la littérature sont un score NIHSS inférieur à 5 , un AVC lacunaire, de petite taille ou intéressant la circulation cérébrale postérieure (cervelet ou tronc cérébral), et la réalisation précoce (dans les 24 premières heures suivant l'apparition des symptômes) de l'IRM cérébrale [5-7]. Chez notre patiente dont l'IRM était normale malgré une lésion significative de localisation cérébrale antérieure et un score NIHSS à 7, nous supposons que c'est la réalisation précoce de l'IRM qui est responsable de ce faux négatif. La réalisation d'une séquence FLAIR lors de l'IRM cérébrale de l'alerte thrombolyse permet de réduire le nombre de faux négatifs : dans l'étude d'Oppenheim et al., quatre patients sur les huit présentant un $\mathrm{AVC}$ ischémique à séquence de diffusion normale avaient une séquence FLAIR pathologique avec des hypersignaux [5]. Les diagnostics différentiels sont les pathologies non vasculaires mimant des AVC, prédominées par l'épilepsie, l'aura migraineuse et les troubles somatoformes [9]. Bien qu'ayant participé à la démarche diagnostique chez notre patiente, l'EEG n'est pas un examen adapté à la détection d'un AVC ischémique à la phase aiguë [10] et n'a d'ailleurs pas sa place dans les recommandations [1].
En conclusion, devant une suspicion clinique d'AVC ischémique, une IRM cérébrale normale ne doit pas éliminer formellement le diagnostic, notamment en présence de facteurs de risque cardiovasculaire. Il semble alors justifié de répéter l'IRM cérébrale pour obtenir la confirmation radiologique du diagnostic, ce qui permet une prise en charge thérapeutique adéquate.

Conflit d'intérêt : les auteurs déclarent ne pas avoir de liens d'intérêts.

\section{Références}

1. Powers WJ, Rabinstein AA, Ackerson T, et al (2018) 2018 guidelines for the early management of patients with acute ischemic stroke: a guideline for healthcare professionals from the American Heart Association/American Stroke Association. Stroke 49: e46-e110

2. Wintermark M, Sanelli PC, Albers GW, et al (2013) Imaging recommendations for acute stroke and transient ischemic attack patients: a joint statement by the American Society of Neuroradiology, the American College of Radiology, and the Society of NeuroInterventional Surgery. Am J Neuroradiol 34:e117-e27

3. Chalela JA, Kidwell CS, Nentwich LM, et al (2007) Magnetic resonance imaging and computed tomography in emergency assessment of patients with suspected acute stroke: a prospective comparison. Lancet 369:293-8

4. Ay H, Buonanno FS, Rordorf G, et al (1999) Normal diffusionweighted MRI during stroke-like deficits. Neurology 52:1784-92

5. Oppenheim C, Stanescu R, Dormont D, et al (2000) Falsenegative diffusion-weighted MR findings in acute ischemic stroke. Am J Neuroradiol 21:1434-40

6. Zuo L, Zhang Y, Xu X, et al (2015) A retrospective analysis of negative diffusion-weighted image results in patients with acute cerebral infarction. Sci Rep 5:8910

7. Tsuyusaki Y, Sakakibara R, Kishi M, et al (2014) "Invisible" brain stem infarction at the first day. J Stroke Cerebrovasc Dis 23:1903-7

8. Morita S, Suzuki M, Iizuka K (2011) False-negative diffusionweighted MRI in acute cerebellar stroke. Auris Nasus Larynx 38:577-82

9. Perot C, Doche E, Laksiri N, et al (2016) Stroke mimics : description des différentes causes et aspects radiologiques rares. Étude sur une cohorte de 192 patients admis en urgence au sein de l'UNV de Marseille. Rev Neurol 172:A75

10. Macdonell RA, Donnan GA, Bladin PF, et al (1988) The electroencephalogram and acute ischemic stroke: distinguishing cortical from lacunar infarction. Arch Neurol 45:520-4 